\title{
The shape effects of nanoparticles suspended in HFE-7100 over wedge with entropy generation and mixed convection
}

\author{
R. Ellahi ${ }^{1,2} \cdot$ M. Hassan ${ }^{2}$ A. Zeeshan ${ }^{2}$ Ambreen A. Khan ${ }^{2}$
}

Received: 7 March 2015 / Accepted: 15 July 2015/Published online: 26 July 2015

(c) The Author(s) 2015. This article is published with open access at Springerlink.com

\begin{abstract}
The flow of mixed convection nanofluid over wedge under the effects of porous medium is investigated. The HFE-7100 Engineered Fluid having Nimonic 80a metal nanoparticles of spherical and non-spherical shapes with different sizes is used. The particle shape effects on Bejan number and entropy generation are taken into account. The system of partial differential equations is first written in terms of ordinary differential equations using adequate similarity transformations and then solved analytically. Analytical solutions of the resulting equations are obtained for the velocity and temperature profiles. Simultaneous effects of porous medium, particle volume friction, mixed convection parameter, and angle of wedge in the presence of different shapes nanoparticles are demonstrated graphically. Effects of particle concentrations, sizes on wall stress, heat transfer coefficient of Skin friction, and Nusselt are discussed in the form of tables.
\end{abstract}

Keywords Nonlinear partial differential equations . Mixed convection - Entropy generation - Shape effects . Nanofluid and porous medium

\section{Introduction}

Nanofluids are a new kind of heat transfer fluid containing a very small quantity of nanoparticles that are stably suspended in a carrier liquid. Fluids have typically very low

R. Ellahi

rellahi@engr.ucr.edu; rahmatellahi@yahoo.com

1 Department of Mechanical Engineering, University of California Riverside, Riverside, USA

2 Department of Mathematics and Statistics, FBAS, IIUI, Islamabad, Pakistan thermal conductivity compared with crystalline solids. However, dispersion with small amount of solid nanoparticles in traditional fluid dramatically changes its thermal conductivities. The problem of heat transfer enhancement was first sorted out by Choi (1995) upon inventing "nanofluid" indicating engineered colloids composed of nanoparticles dispersed in a base fluid. The nanoparticles used in nanofluids are typically made of metals, oxides, carbides, nitrides, and nonmetals. The nanoparticles shapes may be spheres, disks, or rods (Rao 2010). In nanofluid, the base fluid is usually a conductive fluid, such as water or ethylene glycol. Other base fluids are oil and lubricants, bio-fluids, and polymer solutions. The subject of heat transport through dilute suspensions of solid particles has received a renewed boost owing reports of enhancement in thermal conductivity of suspensions carrying nanoparticles. The experimental studies have shown that the enhancement in thermal conductivity of nanofluids is determined by the parameters related to different nanoparticles, concentration, size (Patel et al. 2003; Xie et al. 2002; Chon et al. 2005), shapes (Eastman et al. 2001; Xie et al. 2002, 2003; Choi et al. 2001), agglomeration (fractal-like shapes) (Das et al. 2003; Lee et al. 1999; Xuan and Li 2000; Lee 2007), surface charge (Lee et al. 2006), base fluids, and others. The theory of spherical and non-spherical particles in nanofluid is of great interest in various engineering applications because of their much higher effective thermal conductivities over those of base liquids at very low particle volume concentrations. Poulikakos and coworkers (2010) carefully studied the effect of particle size, concentration, method of stabilization, and clustering on thermal transport in gold nanofluid. A maximum enhancement of $1.4 \%$ was reported for volume $0.11 \%$ suspension of 40-nm-diameter particles in water suggesting no apparent anomaly. Typical thermal conductivity of 
spherical particles enhancements is in the range 15-40\% over the base fluid and heat transfer coefficient enhancements have been found up to $40 \%$ (Yu et al. 2008). In the literature, different authors investigate the thermal properties of cylindrical structures nanoparticle, namely single or multiwall nanotubes suspensions in a variety of solvents (Eastman et al. 2004; Fan and Wang 2011; Keblinski et al. 2008; Kleinstreuer and Feng 2011). Owing to different aspect ratios, solvents, and type of nanotubes (single or multiwall), there is considerable spread in the data. The reported values range from $10 \%$ to over $150 \%$ enhancement in the thermal conductivity for 1 volume $1 \%$ concentration. Singh and coworkers (Singh et al. 2009) studied heat transfer behavior of aqueous suspension of silicon carbide having oblate shape and aspect ratio of around 1/4. They typically observed $30 \%$ enhancement for volume $7 \%$ concentration. Khandekar et al. (2008) employed various spherical nanoparticle as well as Laponite JS (oblate shape, aspect ratio 1/25) based nanofluids in closed two-phase thermosphere and observed its heat transport behavior to be inferior to that of pure water in all cases.

Moreover, porous media are used to transport and store energy in many industrial applications, such as heat exchangers, electronic cooling, heat pipe, solid matrix, and chemical reactors. In particularly, an important characteristic for the combination of the fluid and the porous medium is the tortuosity which represents the hindrance to flow diffusion imposed by local boundaries or local viscosity (Vafai 2011; Vafai and Kim 1989). Furthermore, mixed convection has various applications including cooling system for electronic devices, chemical vapor deposition instrument, furnace engineering, solar energy collectors and building energy system, non-Newtonian chemical processes and domains affected by electromagnetic fields, etc. The convective heat transfer mechanism of nanofluids and porous medium has been the subject of many studies for a better understanding of the associated transport processes. A large number of research work related to the said topics have been conducted by a number of investigators but the studies of mixed convection using nanofluids are very limited. Some relevant studies on the topic can be seen from the list of references (Akbar et al. 2013; Nadeem and Maraj 2014; Nadeem et al. 2013; Sheikholeslami et al. 2014a; b, c, d; Othman et al. 2014; Othman and Zaki 2004; Ibrahim et al. 2009).

Furthermore, a characteristic flow configuration having fundamental importance is that of the flow over a wedge. This type of flow constitutes a general class of investigations in fluid mechanics in which the free stream velocity is proportional to the power of length coordinate measured from the stagnation point. The two-dimensional incompressible wedge flows studied, for the first time by Falkner and Skan (1931). Since then, many authors confined their work in this regime, for instance, Watanabe et al. (1994) have presented the behavior of the thermal boundary layer over a wedge with suction or injection in a mixed convection flow. Heat transfer characteristics in forced convection flow over a wedge subjected to uniform wall heat flux are studied by Yih (1998). Kumari et al. (2001) have investigated the mixed convection flow over a vertical wedge embedded in a highly porous medium, whereas heat transfer analysis on Falkner-Skan wedge flow using differential transformation method is reported by Kuo (2005). The most recent and representative research work, for this type of flow, is presented in Ganapathirao et al. (2013) and several reference can be seen therein.

The core determination of the present work is to examine the shape effects of nanofluid over a wedge by considering the spherical and non-spherical shapes with different sizes of nanoparticles under the influence of nanolayer which has not been yet studied. The HFE-7100, HFE-7200, and HFE-7500 Engineered Fluids which are non-flammable fluid having very low global warming potential in heat transfer applications are used as based fluids. In addition, Bejan number and entropy generation are also taken into account. Due to intrinsic nonlinearity of the governing equations, analytical solutions are very rare. To deal with this difficulty, numerous analytical and semianalytical methods have been established. The "homotopy analysis method" is one of most effective techniques among them to handle this obstacle. In this paper, the solutions of nonlinear resulting equations are carried out using Mathematica package BVPh 2.0 (Zhao and Liao 2013) which is based on homotopy analysis method (HAM). This method is particularly suitable for strongly nonlinear problems (Liao 2003, 2012; Ellahi 2013). The results for velocity, temperature, Bejan number, and Entropy generation are shown in the form of graphs, whereas the tabular results are displayed for heat transfer rate and Skin friction. After the "Introduction" section, the outlines of this paper are as follows. "Mathematical formulation of the problem" section contains mathematical formulation. In "Entropy generation analysis" and "Solution of the problems" sections, Entropy generations analysis and solutions of the problems are presented, respectively. Results and discussion is given in fifth section. Finally, "Conclusions" section summaries the concluding remarks.

\section{Mathematical formulation of the problem}

The steady two-dimensional, incompressible nanofluid flow over a wedge is considered. The wedge is submerged in mixed convective heat transfer and Newtonian fluid (air) flowing with the velocity $u_{\mathrm{e}}(x)=a x^{m}$ in which constant 
$\mathrm{m}=\beta^{*} / 2-\beta^{*}$ (see Kafoussias and Nanousis 1997). If $\beta^{*}<0$, the flow is decelerated, which means that an adverse pressure gradient is imposed, whereas for $\beta^{*}>0$, the flow is accelerated or a favorable pressure gradient is imposed. The fluid on the wedge is subjected to suction or blowing through the entire surface or locally from slots on various locations on the surface of the wedge. The suction/ injection velocity on the wedge surface is $v_{\mathrm{m}}$, whereas the temperature of the surface of the wedge is $T_{\mathrm{w}}(x)$. The HFE7100 engineered fluid is considered as base fluid with Nimonic 80a metal nanoparticles of different shapes for nanofluid.

Under the above assumptions, the equations governing this type of flow can be written in the orthogonal system of coordinates shown in Fig. 1, as follows (Cebeci and Bradshaw 1984; Kafoussias and Xenos 2000). The governing equations for the continuity, momentum, and energy using the Boussinesq and the boundary-layer approximations along with the corresponding boundary conditions can now be written as

$\frac{\partial u}{\partial x}+\frac{\partial v}{\partial y}=0$

$u \frac{\partial u}{\partial x}+v \frac{\partial u}{\partial y}=u_{\mathrm{e}} \frac{\mathrm{d} u_{\mathrm{e}}}{\mathrm{d} x}+v_{\mathrm{nf}} \frac{\partial^{2} u}{\partial y^{2}}+\frac{(\rho \beta)_{\mathrm{nf}}}{\rho_{\mathrm{nf}}} g\left(T-T_{\infty}\right) \sin \Omega$,

$\left(\rho C_{\mathrm{p}}\right)_{\mathrm{nf}}\left(u \frac{\partial T}{\partial x}+v \frac{\partial T}{\partial y}\right)=k_{\mathrm{nf}} \frac{\partial^{2} T}{\partial y^{2}}$,

$\left.u(x, 0)=0, \quad v(x, 0)=v_{\mathrm{w}}(x), \quad u(x, \infty)=u_{\mathrm{e}}(x)\right\}$

$\left.T(x, 0)=T_{\mathrm{w}}(x)=T_{\infty}+C x^{m}, \quad T(x, \infty)=T_{\infty}.\right\}$,

where $u$ and $v$ are the velocity components in the $x$ - and $y$ directions, respectively.

The suction/injection velocity distribution across the wedge surface is assumed to have the following function:

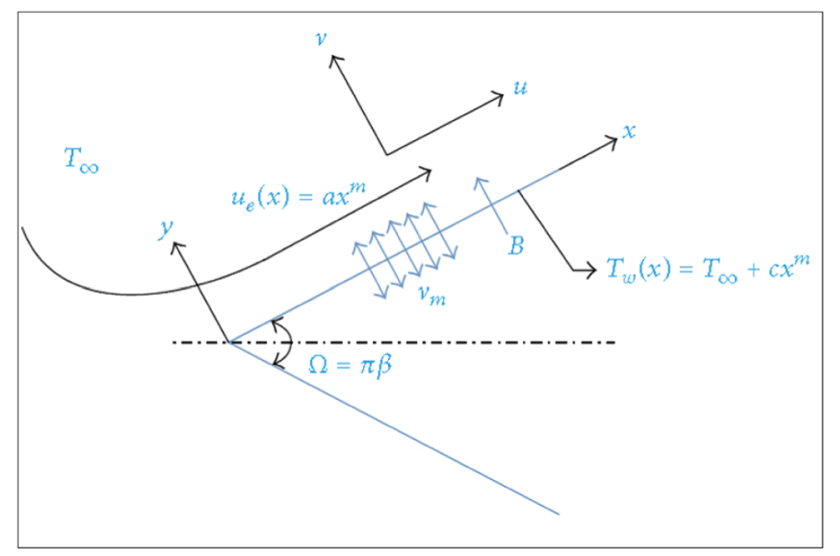

Fig. 1 Configuration of the flow and geometrical coordinates $v_{\mathrm{w}}=-f_{\mathrm{w}}(v a)^{1 / 2}((m+1) / 2) x^{(m-1) / 2}$,

where $v_{\mathrm{w}}$ is the dimensionless wall mass transfer coefficient. When we choose $v_{\mathrm{w}}>0$ velocity across the surface of the boundary wall, it shows the mass injection, and when chooses velocity of mass $v_{\mathrm{w}}<0$, it shows mass suction process. The total angle of wedge is $\Omega=\pi \beta^{*} . T_{\mathrm{w}}$ and $T_{\infty}$ is the temperature of wall and temperature of the ambient fluid. In Eqs. (1)-(3), the effective density $\rho_{\mathrm{nf}}$, heat capacitance $\left(\rho C_{\mathrm{p}}\right)_{\mathrm{nf}}$, thermal expansion coefficient $\beta_{\mathrm{nf}}$, and thermal diffusibility $\alpha_{\text {nf }}$ of the nanofluid are defined by

$\rho_{\mathrm{nf}}=(1-\phi) \rho_{\mathrm{f}}+\phi \rho_{\mathrm{s}}$,

$\left(\rho C_{\mathrm{p}}\right)_{\mathrm{nf}}=(1-\phi)\left(\rho C_{\mathrm{p}}\right)_{\mathrm{f}}+\phi\left(\rho C_{\mathrm{p}}\right)_{\mathrm{s}}$,

$\beta_{\mathrm{nf}}=\frac{(1-\phi)(\rho \beta)_{\mathrm{f}}+\phi(\rho \beta)_{\mathrm{s}}}{\rho_{\mathrm{nf}}}$,

$\alpha_{\mathrm{nf}}=\frac{k_{\mathrm{nf}}}{\left(\rho C_{\mathrm{p}}\right)_{\mathrm{nf}}}$

Here, $\phi$ is the solid volume friction, $\beta_{\mathrm{f}}$ and $\beta_{\mathrm{s}}$ are respectively the thermal expansion coefficients of the base fluid and nanoparticle, $\rho_{\mathrm{f}}$ and $\rho_{\mathrm{s}}$ are the densities of basic fluid and nanoparticle, respectively. We will use the thermal conductive model for nanoparticles for spherical and non-spherical (Yu and Choi 2004) as follows:

$k_{\mathrm{nf}}=\left(1+\frac{m \phi_{\mathrm{e}} A}{1-\phi_{\mathrm{e}} A}\right) k_{\mathrm{f}}$,

in which the parameter $A$ is given by

$A=\frac{1}{3} \sum_{j=a, b, c} \frac{k_{\mathrm{p} j}-k_{f}}{k_{\mathrm{p} j}+\left(n^{*}-1\right) k_{\mathrm{f}}}$.

In above Eq. (10), $n^{*}=3 / \psi$ is the empirical shape factor $(\psi$ is the sphericity defined as ratio between the surface area of the sphere and the surface area of the real particle with equal volumes), $\phi_{\mathrm{e}}$ is particle volume friction with nanolayer of base fluid on particle, $k_{\mathrm{f}}$ is the thermal conductivity of base fluid, and $k_{\mathrm{p} j}$ id thermal conductivity along the axes of the particle which is defined by

$k_{\mathrm{p} j}=\left[1+\frac{k_{\mathrm{p}}-k_{1}}{k_{\mathrm{p}}\left[r_{1} d(j, 0)-d(j, t)\right]-k_{1}\left[r_{1} d(j, 0)-d(j, t)-r_{1}\right]}\right] k_{\mathrm{s}}$,

where $j(=a, b$, and $c)$ is along the semi-axes direction of the particle, whereas $k_{\mathrm{p}}$ and $k_{1}$ are the thermal conductivities of the solid particle and its surrounding layer, $r_{1}$ is the volume ratio and $d(j, v)$ is depolarization factor defined by

$r_{1}=\frac{\sqrt{\left(a^{2}+t\right)\left(b^{2}+t\right)\left(c^{2}+t\right)}}{a b c}$, 


$$
\begin{aligned}
d(j, v)= & \frac{\sqrt{\left(a^{2}+v\right)\left(b^{2}+v\right)\left(c^{2}+v\right)}}{2} \\
& \times \int_{0}^{\infty} \frac{\mathrm{d} w}{\left(j^{2}+v+w\right) \sqrt{\left(a^{2}+v+w\right)\left(b^{2}+v+w\right)\left(c^{2}+v+w\right)}},
\end{aligned}
$$

with $v=0$ for outside of solid ellipsoid and $v=t$ for outside surface of its surrounding layer. For a needleshaped particle with $a=b \gg c, d(a, v), d(b, v)$ and $d(c, v)$ respectively tend to $0,1 / 2$, and $1 / 2$ whereas for a disk-shaped particle $d(a, v), d(b, v)$, and $d(c, v)$ respectively tend to 0,0 , and 1 , provided that $a=b \gg c$. For a sphere with $a=b=c$, the three factors are $1 / 3$. The effective viscosity models (Timofeeva et al. 2009) for nanofluid are given by as follows:

$\mu_{\mathrm{nf}}=1+A_{1} \phi+A_{2} \phi^{2}$.

In the thermal conductive and viscosity models, the value of empirical shape factor $n^{*}$, sphericity $\psi$, the values of $A_{1}$ and $A_{2}$ is shown in Table 1.

Physical properties of base fluid and particles are shown in Table 2.

In order to solve the system of PDEs, the compressible version of the Falkner-Skan transformation for a wedge (Kafoussias and Nanousis 1997) is introduced, defined by

$$
\left.\begin{array}{c}
\eta=\sqrt{\frac{u_{\mathrm{e}}(x)}{v_{f} x}} y, \quad \psi=\sqrt{v_{\mathrm{f}} x u_{\mathrm{e}}(x)} f(\eta), \\
\theta(\eta)=\frac{T-T_{\infty}}{T_{\mathrm{w}}-T_{\infty}}
\end{array}\right\},
$$

where $\psi(x, y)$ satisfies the continuity equation.

The stream function is defined as

$u=\frac{\partial \psi}{\partial y} \quad$ and $\quad v=-\frac{\partial \psi}{\partial x}$.

Table 1 The value of parameters for thermal conductive and viscosity models

\begin{tabular}{lllll}
\hline Nanoparticles & Sphericity & Shape factor $\left(n^{*}\right)$ & $A_{1}$ & \multicolumn{1}{c}{$A_{2}$} \\
\hline Needle-shaped & 0.62 & 4.9 & 13.5 & 904.4 \\
Disk-shaped & 0.36 & 8.6 & 14.6 & 123.3 \\
Sphere-shaped & 1 & 3 & 2.5 & 6.2 \\
\hline
\end{tabular}

Table 2 Physical properties of base fluid and particles for nanofluid \begin{tabular}{lllll}
\hline Nanoparticles & HFE-7100 HFE-7200 & HFE-7500 Nimonic 80a
\end{tabular}

\begin{tabular}{lllll}
\hline$\rho\left(\mathrm{kg} / \mathrm{m}^{3}\right)$ & 1510 & 1430 & 1610 & 8190 \\
$C_{\mathrm{p}}(\mathrm{J} / \mathrm{kg}-\mathrm{K})$ & 1180 & 1220 & 1130 & 448 \\
$k(\mathrm{~W} / \mathrm{m}-\mathrm{K})$ & 0.0678 & 0.07 & 0.065 & 112 \\
$\beta(1 / \mathrm{K})$ & 0.0018 & 0.0016 & 0.0013 & 0.0000127 \\
\hline
\end{tabular}

Substituting into Eqs. (1)-(4), one can get the nondimensional form of Eqs. (16) and (17) along with associated boundary conditions

$\frac{\rho_{\mathrm{nf}}}{\rho_{\mathrm{f}}}\left\{m f^{\prime 2}-\left(\frac{m+1}{2}\right) f^{\prime \prime}\right\}=m+\frac{\mu_{\mathrm{nf}}}{\mu_{\mathrm{f}}} f^{\prime \prime \prime}+\lambda \sin (\Omega / 2) \frac{(\rho \beta)_{\mathrm{nf}}}{(\rho \beta)_{\mathrm{f}}} \theta$,

$\operatorname{Pr} \frac{\left(\rho C_{\mathrm{p}}\right)_{\mathrm{nf}}}{\left(\rho C_{\mathrm{p}}\right)_{\mathrm{f}}}\left\{m f^{\prime} \theta-\left(\frac{m+1}{2}\right) f \theta^{\prime}\right\}=\frac{k_{\mathrm{nf}}}{k_{f}}\left(\frac{m+1}{2}\right) \theta^{\prime \prime}$,

$\left.\begin{array}{r}f(0)=f_{\mathrm{w}}, \quad f^{\prime}(0)=0, \quad f^{\prime}(\infty)=1 \\ \theta(0)=1, \quad \theta(\infty)=0\end{array}\right\}$,

in which

$$
\begin{aligned}
& \operatorname{Pr}=\frac{v_{\mathrm{f}}}{\alpha_{\mathrm{f}}}, \quad \lambda=\frac{G r}{\operatorname{Re}^{2}}, \quad G r=\frac{\beta_{\mathrm{f}} g\left(T_{\mathrm{w}}-T_{\infty}\right) x^{3}}{v_{\mathrm{f}}^{2}}, \\
& \operatorname{Re}=\frac{u_{\mathrm{e}} x}{v_{\mathrm{f}}}
\end{aligned}
$$

where $\operatorname{Pr}$ is the Prandtl number, $\lambda$ is the mixed convection parameter, $G r$ is the Grashof number, and $R e$ is the Reynolds numbers.

The wall shear stress (Skin friction coefficient) can be expressed in demission less form as

$C_{\mathrm{f}} \operatorname{Re}_{x}^{1 / 2}=2\left(\frac{\mu_{\mathrm{nf}}}{\mu_{\mathrm{f}}}\right) f^{\prime \prime}(0)$.

The local Nusselt number (heat transfer rate) is obtained in dimensionless form as follows:

$N u \mathrm{Re}_{x}^{-1 / 2}=-\frac{k_{\mathrm{nf}}}{k_{\mathrm{f}}} \theta^{\prime}(0)$.

\section{Entropy generation analysis}

The local volumetric rate of entropy generation for a nanofluid flow (Butt and Ali 2013) is defined by

$$
S_{\text {gen }}=\underbrace{\frac{k_{\mathrm{nf}}}{T_{\infty}^{2}}\left(\frac{\partial T}{\partial y}\right)^{2}}_{\text {HFI }}+\underbrace{\frac{\mu_{\mathrm{nf}}}{T_{\infty}}\left(\frac{\partial u}{\partial y}\right)^{2}}_{\text {FFI }} .
$$

In Eq. (16), the first term HFI is the entropy generation due to heat transfer and the second term FFI is the entropy generation due to viscous dissipation. The dimensionless form of the entropy generation is given by

$N_{\mathrm{s}}=\frac{S_{\text {gen }}}{S_{\mathrm{o}}}=\frac{k_{\mathrm{nf}}}{k_{\mathrm{f}}} R_{\mathrm{e}} \theta^{\prime 2}+\frac{\mu_{\mathrm{nf}}}{\mu_{\mathrm{f}}} \frac{R_{\mathrm{e}} B r}{\omega} f^{\prime \prime 2}$,

where $S_{\mathrm{o}}=k_{\mathrm{f}}\left(T_{\mathrm{w}}-T_{\infty}\right)^{2} / T_{\infty}^{2} x^{2}$ is the characteristic entropy generation rate, $\omega=\frac{T_{\infty}}{T_{\mathrm{w}}-T_{\infty}}$ is the dimensionless temperature difference, $B r=\operatorname{Pr} E c$ is the Brinkman 
number. In which, $T_{\mathrm{w}}$ and $T_{\infty}$ are measured in degree of Kelvin.

An alternative irreversibility distribution parameter is the Bejan number $B e$, which gives an idea whether the fluid friction irreversibility dominates over heat transfer irreversibility or the heat transfer irreversibility dominates over fluid friction irreversibility. It is simply the ratio of entropy generation due to heat transfer to the total entropy generation

$$
B e=\frac{\mathrm{HFI}}{\mathrm{HFI}+\mathrm{FFI}} .
$$

when $B e \gg 0.5$, the irreversibility due to heat transfer dominates, whereas when $B e \ll 0.5$, the irreversibility due to viscous effects dominates. When $B e=0.5$, the heat transfer and the fluid friction irreversibility are equal.

\section{Solution of the problems}

In this section, the analytical solutions of Eqs. (16) and (17) will be determined for the velocity and temperature profiles by using Mathematica package BVPh 2.0 which is based on the HAM. For simplicity, the BVPh 2.0 needs to input the governing equations along with corresponding boundary conditions and choose proper initial guess of solutions and auxiliary linear operators for under consideration linear sub-problems. In this package, one has great freedom to choose the auxiliary linear operator and initial guess, thus we choose the auxiliary linear operators and initial guess for the desire solutions as follows:

$f_{0}(\eta)=-1+f_{w}+\eta+e^{-\eta}, \quad \theta_{0}(\eta)=e^{-\eta}$

as the initial approximation of $f$ and $\theta$, respectively, which satisfy the following linear operator and corresponding boundary conditions:

$£_{1}(f)=\left(\frac{\mathrm{d}^{3}}{\mathrm{~d} \eta^{3}}-\frac{\mathrm{d}}{\mathrm{d} \eta}\right) f, \quad £_{2}(\theta)=\left(\frac{\mathrm{d}^{2}}{\mathrm{~d} \eta^{2}}-1\right) \theta$.

Using the linear auxiliary operators in Eq. (25) and the initial approximations in Eq. (24), the coupled nonlinear Eqs. (16) and (17) subject to the boundary conditions given in Eq. (18), finally the solutions of velocity and temperature distributions can be expressed explicitly by an infinite series of the following form:

$$
\begin{aligned}
& f_{i}(\eta)=\sum_{m=0}^{\infty} f_{i, m}(\eta), \\
& \theta_{i}(\eta)=\sum_{m=0}^{\infty} \theta_{i, m}(\eta),
\end{aligned}
$$

where $f_{i, m}(\eta)$ and $\theta_{i, m}(\eta)$ are governed by high-order deformation equations, which are linear and dependent upon auxiliary linear operators given in Eq. (25). We get the results for velocity, temperature distribution, Sink fiction, and Nusselt numbers for different non-dimensional numbers at 30th iterations of package.

The solutions for velocity and temperature up to second iteration are as follows:

$$
\begin{aligned}
f(\eta)= & 1+\left(\begin{array}{c}
-1-\frac{11 A_{1}}{30}+\frac{187 A_{2}}{1440}+\frac{209 A_{2} m}{288} \\
+\frac{11 A_{2} f_{\mathrm{w}}}{60}+\frac{11 m \eta}{20}-\frac{11 A_{2} m \eta}{20}+\frac{11 A_{3} \lambda}{30} \sin (\Omega / 2)
\end{array}\right) e^{-\eta} \\
& +\left(\begin{array}{c}
\frac{11 A_{1}}{30}-\frac{11 A_{2}}{180}-\frac{143 A_{2} m}{180} \\
-\frac{11 A_{2} \eta}{60}-\frac{11 A_{2} \eta f_{\mathrm{w}}}{60}-\frac{11 A_{2} m \eta}{60}-\frac{13 A_{3} \lambda}{30} \sin (\Omega / 2)
\end{array}\right) e^{-2 \eta} \\
& +\left(\begin{array}{c}
\frac{-11 A_{2}}{160} \\
+\frac{11 A_{2} m}{160}
\end{array}\right) e^{-3 \eta},
\end{aligned}
$$

$$
\begin{aligned}
& \theta(\eta)=\left(\begin{array}{c}
1+\frac{13 B_{1}}{30}-\frac{221 B_{2} P r}{1440}+\frac{13 A_{1} E c P r}{60} \\
-\frac{611 B_{2} m P r}{1440}-\frac{611 B_{2} \operatorname{Pr} f_{\mathrm{w}}}{60}+\frac{13 B_{2} m \operatorname{Pr} f_{\mathrm{w}}}{60}
\end{array}\right) e^{-\eta} \\
&+\left(\begin{array}{c}
-\frac{3 B_{1}}{30}+\frac{13 B_{2} \operatorname{Pr}}{180}+\frac{91 B_{2} m P r}{180} \\
+\frac{13 B_{2} \operatorname{Pr} f_{\mathrm{w}}}{60}+\frac{13 B_{2} m \operatorname{Pr} f_{\mathrm{w}}}{60} \\
+\frac{13 B_{2} \operatorname{Pr} \eta}{60}+\frac{13 B_{2} m P r \eta}{60}
\end{array}\right) e^{-2 \eta} \\
&+\left(\begin{array}{c}
\frac{13 B_{1}}{160}-\frac{13 A_{1} E c \operatorname{Pr}}{60} \\
-\frac{13 B_{2} m P r}{160}
\end{array}\right) e^{-3 \eta},
\end{aligned}
$$

where

$$
\begin{gathered}
A_{1}=\frac{\mu_{\mathrm{nf}}}{\mu_{\mathrm{f}}}, \quad A_{2}=\frac{\rho_{\mathrm{nf}}}{\rho_{\mathrm{f}}}, \quad A_{3}=\frac{(\rho \beta)_{\mathrm{nf}}}{(\rho \beta)_{\mathrm{f}}}, \quad B_{1}=\frac{k_{\mathrm{nf}}}{k_{\mathrm{f}}}, \\
B_{2}=\frac{\left(\rho C_{\mathrm{p}}\right)_{\mathrm{nf}}}{\left(\rho C_{\mathrm{p}}\right)_{\mathrm{nf}}} .
\end{gathered}
$$

\section{Results and discussion}

In this section, the behavior of emerging parameters involved in the expression of velocity and temperature distributions is examined through Figs. 2, 3, 4, 5, 6, 7, 8, 9 , 10 , and 11 with HEF-7100 based nanofluids contained Nimonic 80a nanoparticles. The governing equation and their boundary conditions are transferred to ordinary differential equations. These equations include the nanoparticles volume friction $\varphi$, mixed convection parameter $\lambda$, suction or injection parameter $f_{\mathrm{w}}$, and total angle of wedge is $\Omega$. In this study, for velocity and temperature profile, we 


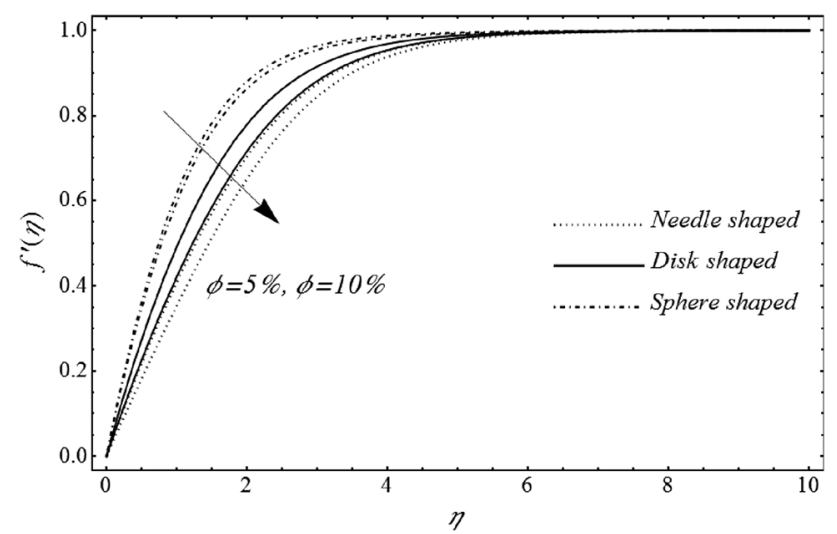

Fig. 2 Effect of volume friction on velocity profile

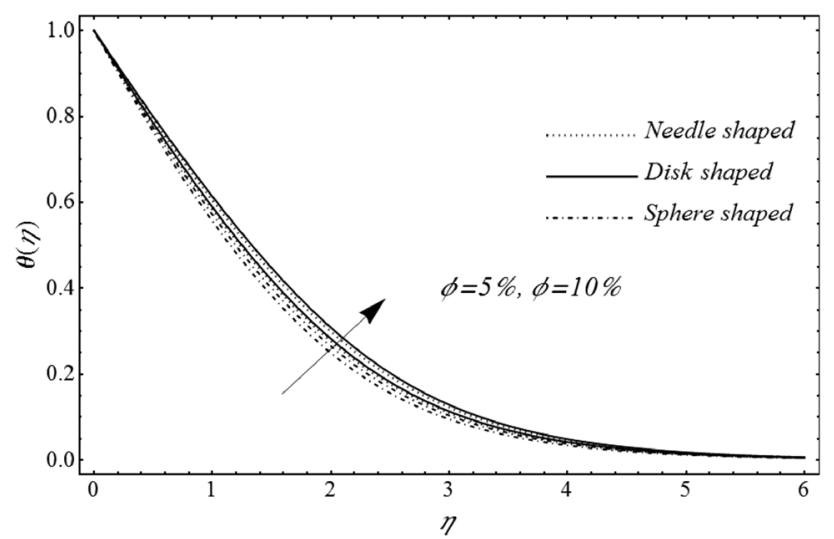

Fig. 3 Effect volume friction on temperature profile

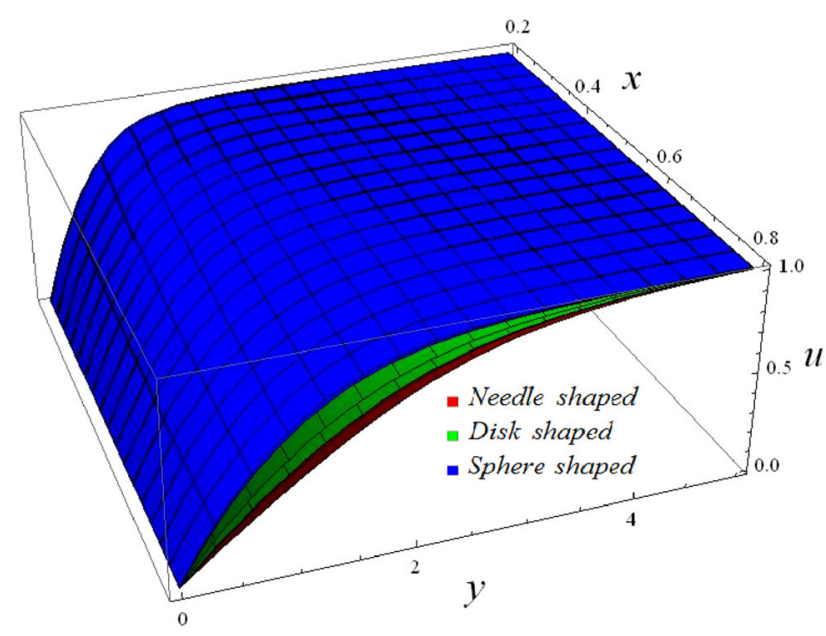

Fig. 4 Effect of volume friction on velocity profile

prepare the particles of different shapes by applying the Eq. (10). We take spherical shape particle of $5 \mathrm{~nm}$ radius, needle shape of $5 \mathrm{~nm}$ radius and $50 \mathrm{~nm}$ length and disk shape of $50 \mathrm{~nm}$ radius and thickness of $5 \mathrm{~nm}$. We also

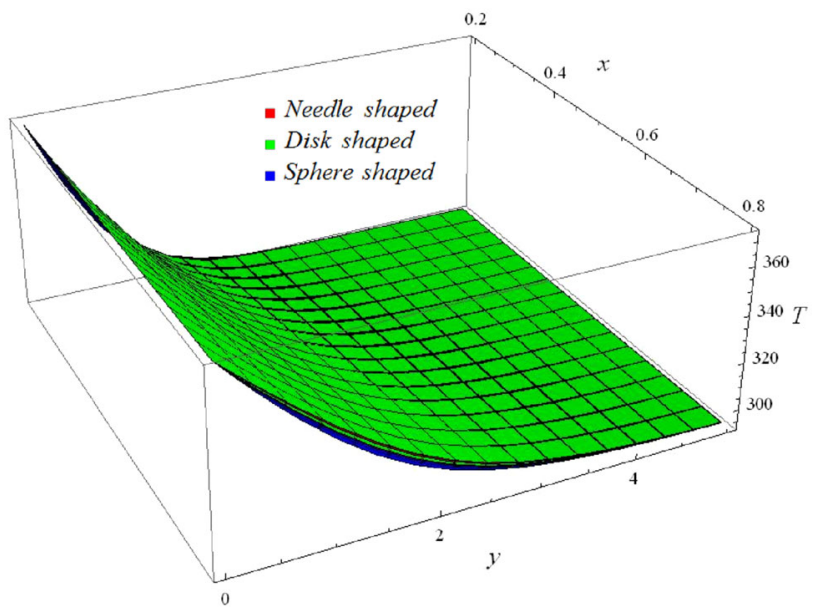

Fig. 5 Effect of volume friction on temperature profile

consider nanolayer thickness $2 \mathrm{~nm}$ of base fluid on particle and for this take the value $t=24$. The thermal conductive of nanolayer $k_{1}$ is consider $2 k_{\mathrm{f}}$. These figures $0 \% \leq \phi \leq 10 \%$ are prepared within range of particle volume friction and consider that heat is dominant in fluid. The effects of particle volume friction on velocity and temperature profiles are shown in Figs. 2 and 3, respectively. Figure 2 points out the velocity of fluid decreases by increasing the particle concentration. It is due to the fact that when particle concentrations become enlarge, the viscosity of fluid is enhanced. It is in accordance with the physical expectation since the fluid having low viscosity can easily move as compared to high viscosity fluid. It is also observed that the maximum velocity decreases for needle followed by disk and sphere shapes particles, respectively. On the other hand, in Fig. 3, the temperature profile increases for raising the particle volume friction. This is in accordance with the fact that temperature of fluid becomes enlarged when the thermal conductivity is elevated and as a result the thermal conductivity increases by increasing the particle volume friction. It is noticed that the maximum temperature of nanofluid increases for diskshaped followed by the needle-shaped and sphere-shaped particles, respectively. Figures 4 and 5 show the effects of volume friction on velocity and temperature profile. To see the 3D impact, these figures are prepared for back replacing the transformations $T_{\infty}=295 \mathrm{~K}$ and $\Delta T=80 \mathrm{~K}$. Figures 6 and 7 show the behavior of non-dimensional mixed convection parameter $\lambda$ on velocity and temperature profile of nanofluid. This parameter is ratio of buoyancy forces to the inertial forces inside the boundary layer for the laminar boundary layer forced-free convective flows. However, forced convection exists when the limit of $\lambda$ tends to zero and the free convection limit can be reached if it becomes large. These figures depict that when the buoyancy force is increased, the velocity of the fluid is 


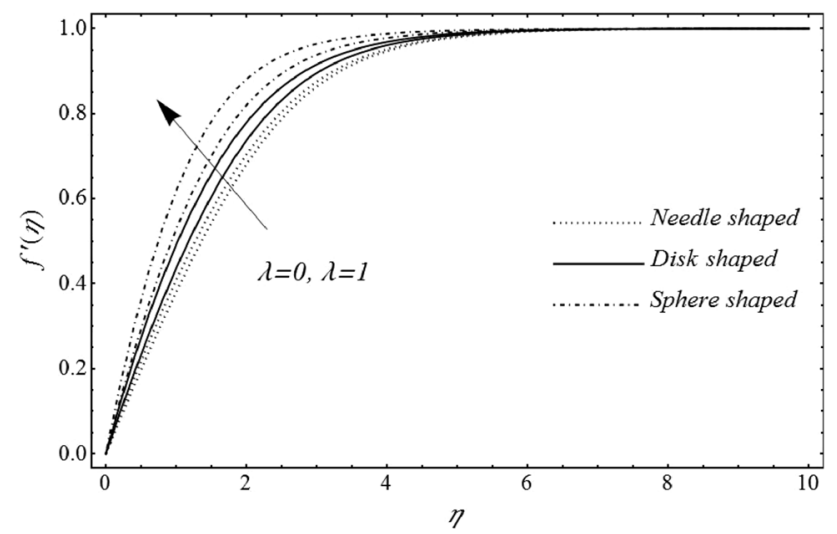

Fig. 6 Effect of mixed convection parameter on velocity profile

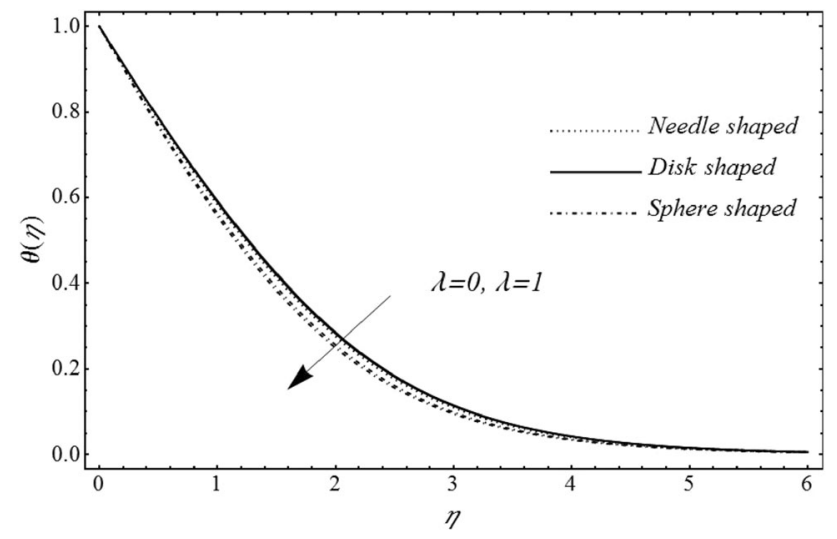

Fig. 7 Effect of mixed convection parameter on temperature profile

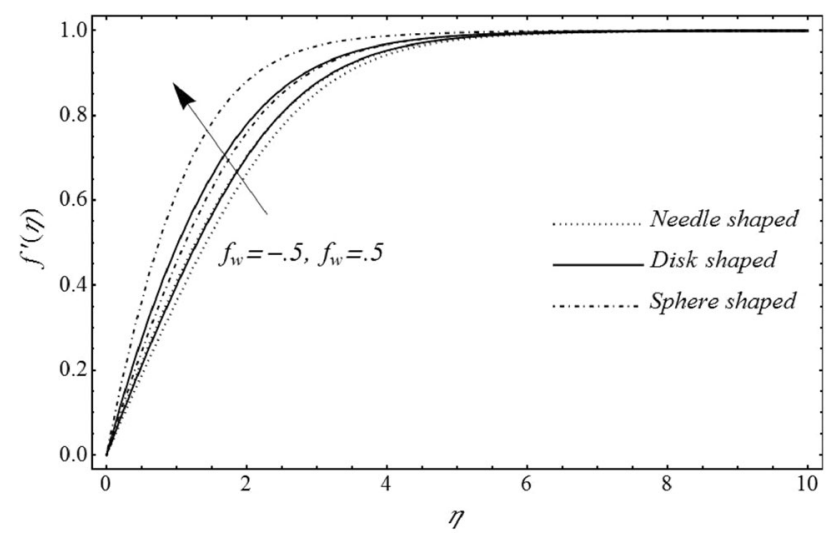

Fig. 8 Effect of suction or injection parameter on velocity profile

enhanced, whereas temperature is increased when boundary layer flow turn to free to force convection. The effects of suction or injection parameter on velocity and temperature are plotted in Figs. 8 and 9. When one chooses $v_{\mathrm{w}}>0$, the velocity across the surface of the boundary wall, it means $f_{\mathrm{w}}<0$ that is mass injection. When one chooses velocity of mass $v_{\mathrm{w}}<0$, it means $f_{\mathrm{w}}>0$ which

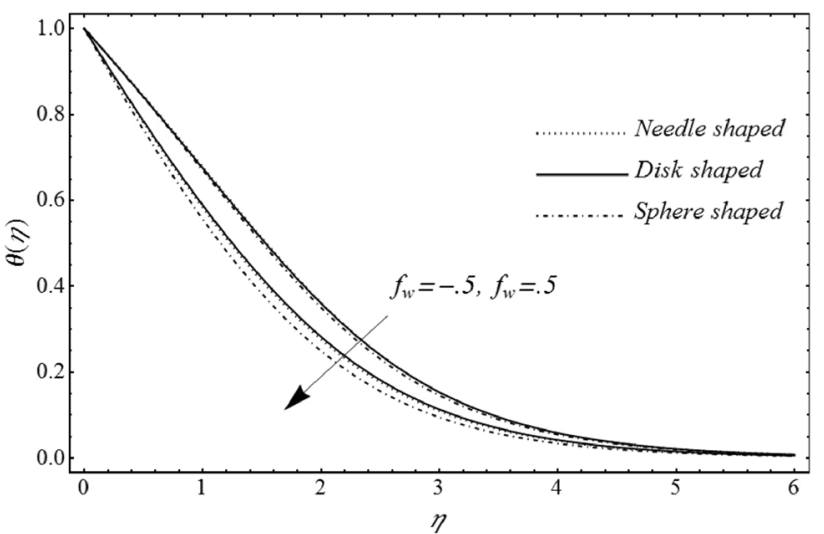

Fig. 9 Effect of suction or injection parameter on temperature profile

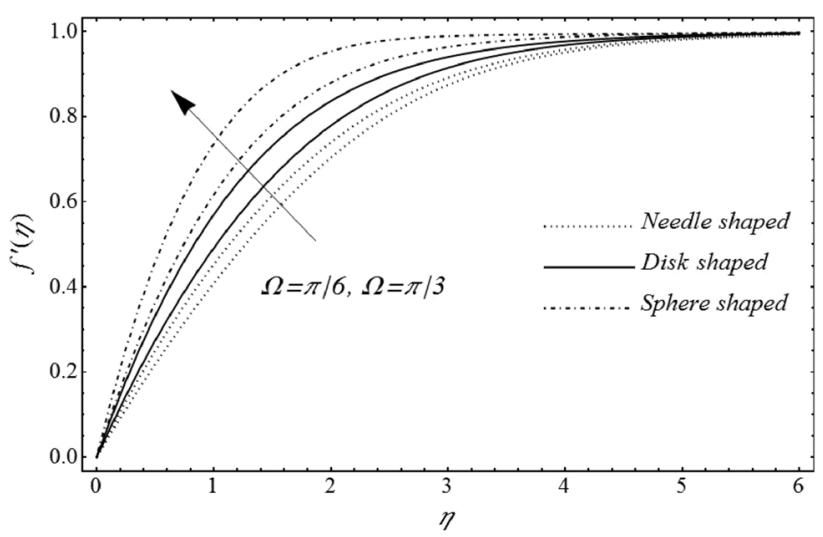

Fig. 10 Effect of total angle of wedge on velocity profile

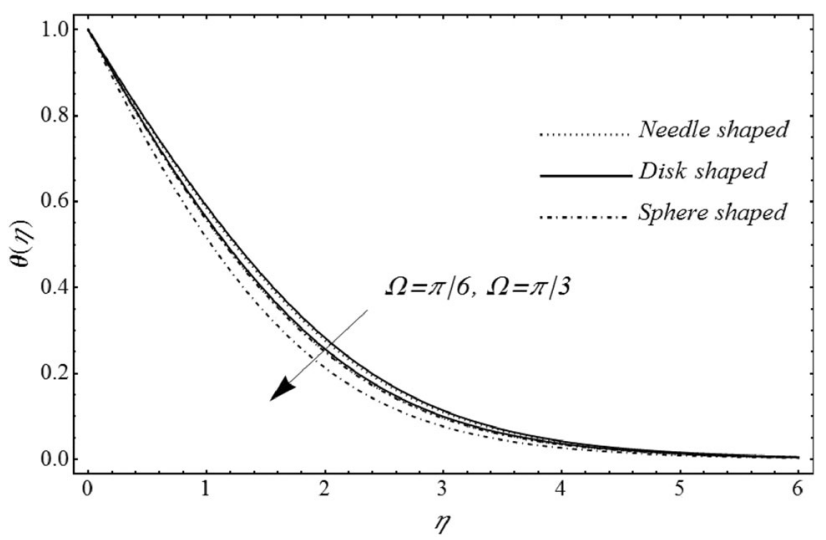

Fig. 11 Effect of total angle of wedge on temperature profile

shows mass suction. Figure 8 demonstrates the enhancement in velocity distribution in boundary layer region for the suction case. In Fig. 9, it is observed that the temperature is reduced when the value of $f_{\mathrm{w}}$ is enlarged. Figures 10 and 11 illustrate the effects of angle of wedge on the non-dimensional velocity and temperature in the presence of spherical and non-spherical nanoparticles. As it is 


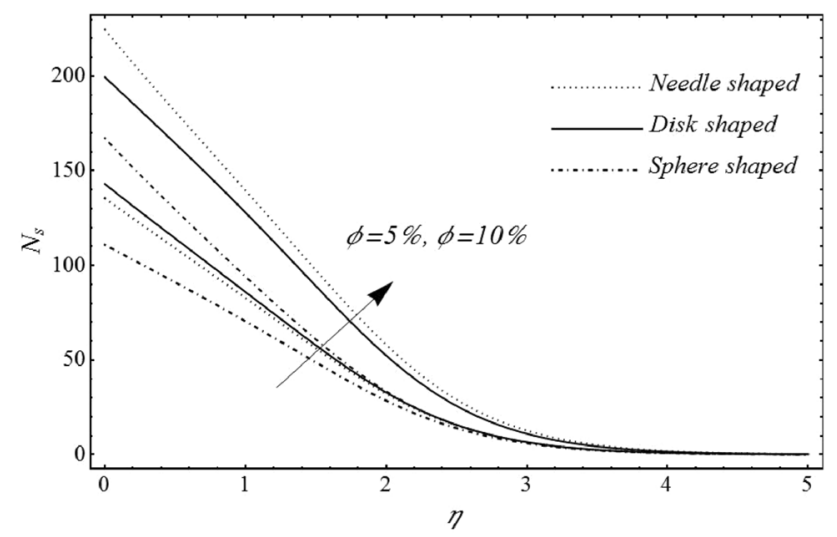

Fig. 12 Effect of volume friction on entropy generation number

seen that when angle of wedge increases, the velocity profile is decreased as shown in Fig. 10. In addition, when angle of wedge increases, the temperature of nanofluid is declined.

The effects of the particles volume friction on entropy generation number Ns are shown in Fig. 12. It is noticed from this figure that entropy production increases by increasing the particles volume friction. It is also observed that the irreversibility near the surface has maximum and then decreases and approaches to zero far from the disk. The maximum irreversibility is realized by disk-shaped particles when compared with other shapes (Fig. 13).

The numerically results in Tables $3,4,5,6$, and 7 illustrate the effects of various parameters on the skin friction coefficient, heat transfer rate of spherical and nonspherical nanoparticles suspended in HEF-7100 fluid. These tables are prepared by fixing the parameters $\lambda=2$, $f_{\mathrm{w}}=2$, and $\Omega=\pi / 6$. The effects of particle volume friction on the local skin friction coefficients and local Nusselt number are shown in Table 3. Table 3 depicts that with
Table 3 Effect of volume friction on skin friction coefficient and Nusselt number

\begin{tabular}{llll}
\hline$\phi(\%)$ & Needle & Disk & Sphere \\
\hline$C_{\mathrm{f}} \operatorname{Re}_{x}^{1 / 2}$ & & & \\
0 & 1.8282 & 1.8282 & 1.8282 \\
2 & 2.1926 & 2.0504 & 1.8927 \\
4 & 2.9362 & 2.3162 & 1.9589 \\
$N u \operatorname{Re}_{x}^{1 / 2}$ & & & \\
0 & 0.5162 & 0.5162 & 0.5162 \\
2 & 0.5296 & 0.5581 & 0.5402 \\
4 & 0.5477 & 0.6047 & 0.5649 \\
\hline
\end{tabular}

Table 4 Effect of dimension of needle on skin friction coefficient and Nusselt number

\begin{tabular}{|c|c|}
\hline Dimension & Needle \\
\hline \multicolumn{2}{|l|}{$C_{\mathrm{f}} R e_{x}^{1 / 2}$} \\
\hline$a=25, b=c=2.5$ & 3.4633 \\
\hline$a=50, b=c=5$ & 3.4601 \\
\hline$a=75, b=c=10$ & 3.4586 \\
\hline \multicolumn{2}{|l|}{$N u R e_{x}^{1 / 2}$} \\
\hline$a=25, b=c=2.5$ & 0.5763 \\
\hline$a=50, b=c=5$ & 0.5607 \\
\hline$a=75, b=c=10$ & 0.5542 \\
\hline
\end{tabular}

increasing the volume friction of nanoparticles suspension in the base fluid, both local skin friction coefficients and heat transfer rate increase, respectively. The maximum wall shear stress is caused by needle-shaped particle. Moreover, the maximum heat transfer rate is due to the disk-shaped particle. In addition, the heat transfer rate of HFE-7100 based nanofluid is improved 8.3, 24, and 13.3 corresponding to needle-shaped, disk-shaped, and sphere-

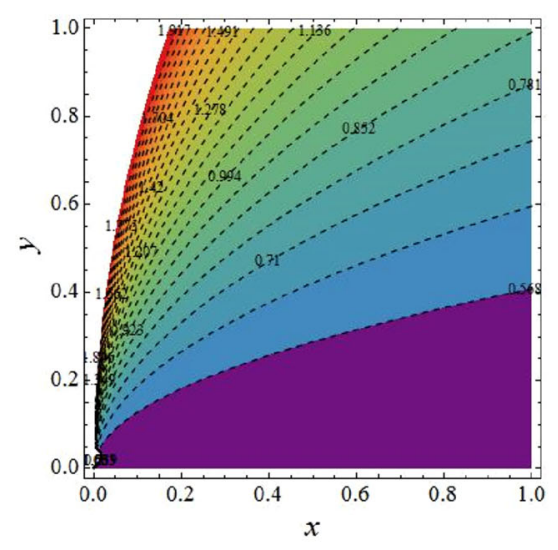

(a)

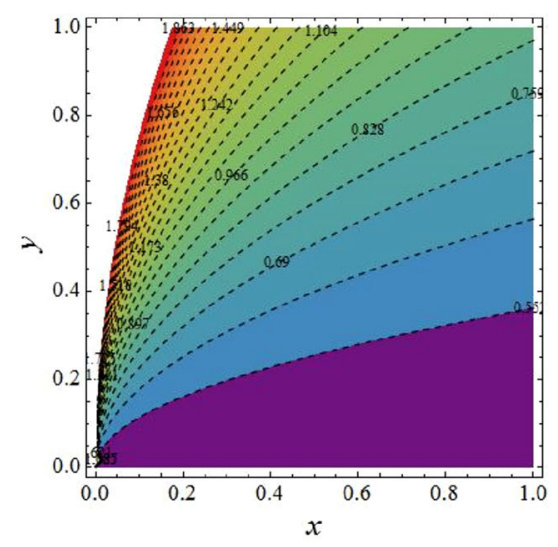

(b)

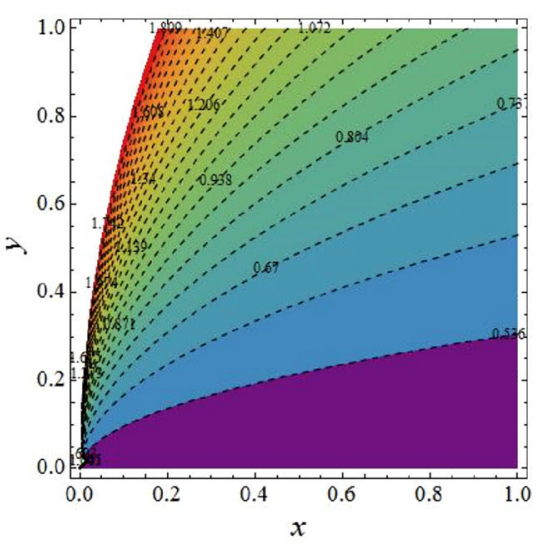

(c)

Fig. 13 Effects of particle volume friction on stream lines over a wedge. a Stream lines when $\phi=0 \%$, b stream lines when $\phi=5 \%$, and c stream lines when $\phi=10 \%$ 
Table 5 Effect of dimension of disk on skin friction coefficient and Nusselt number

\begin{tabular}{|c|c|}
\hline Dimension & Disk \\
\hline \multicolumn{2}{|l|}{$C_{\mathrm{f}} R e_{x}^{1 / 2}$} \\
\hline$a=b=25, c=2.5$ & 2.4718 \\
\hline$a=b=50, c=5$ & 2.4670 \\
\hline$a=b=75, c=10$ & 2.4651 \\
\hline \multicolumn{2}{|l|}{$N u R e_{x}^{1 / 2}$} \\
\hline$a=b=25, c=2.5$ & 0.6585 \\
\hline$a=b=50, c=5$ & 0.6297 \\
\hline$a=b=75, c=10$ & 0.6196 \\
\hline
\end{tabular}

Table 6 Effect of dimension of sphere on skin friction coefficient and Nusselt number

\begin{tabular}{|c|c|}
\hline Dimension & Sphere \\
\hline \multicolumn{2}{|l|}{$C_{\mathrm{f}} R e_{x}^{1 / 2}$} \\
\hline$a=b=c=2.5$ & 1.9813 \\
\hline$a=b=c=5$ & 1.9928 \\
\hline$a=b=c=10$ & 1.9927 \\
\hline \multicolumn{2}{|l|}{$N u R e_{x}^{1 / 2}$} \\
\hline$a=b=c=2.5$ & 0.5425 \\
\hline$a=b=c=5$ & 0.5776 \\
\hline$a=b=c=10$ & 0.5773 \\
\hline
\end{tabular}

Table 7 The values of skin friction coefficient and Nusselt number for different base fluids

\begin{tabular}{llll}
\hline Base fluids & Needle & Disk & Sphere \\
\hline$C_{\mathrm{f}} \operatorname{Re}_{x}^{1 / 2}$ & & & \\
HFE-7100 & 3.4601 & 2.4670 & 1.9928 \\
HFE-7200 & 3.4661 & 2.4738 & 1.9999 \\
HFE-7500 & 3.4601 & 2.4599 & 1.9854 \\
$N u \operatorname{Re}_{x}^{1 / 2}$ & & & \\
HFE-7100 & 0.5607 & 0.6297 & 0.5776 \\
HFE-7200 & 0.5612 & 0.6304 & 0.5784 \\
HFE-7500 & 0.5607 & 0.6292 & 0.5768 \\
\hline
\end{tabular}

shaped particle at $6 \%$ of particle volume friction. The effects of particle's size on skin friction coefficient and heat transfer are shown in Tables 4, 5, and 6. It is observed that the local skin friction as well as local Nusselt number decreases with the enhancement in length, radius of needleshaped particles, thickness of disk-shaped particles and radius of sphere-shaped particles. It is also seen that the small size particles are more effective for heat transfer rate as compared to large size particle. For example, when one take spherical particle, the heat transfer rate is improved 11 and $10 \%$ corresponding to radius of 5 and $30 \mathrm{~nm}$ at $5 \%$ particle volume friction, respectively. To investigate wall shear stress and heat transfer rate, three different Novecbased fluids are examined in Table 7. It is found that the effect of wall shear stress maximum reduces by HFE-7500 fluid than other, while maximum enhanced in heat transfer rate is observed for HFE-7200 fluid as compared to rest of Novec fluids.

\section{Conclusions}

In this paper, the shape effect on HFE-7100 based nanofluid with influence of different parameters is investigated. It is observed that the velocity of nanofluid decreases by increasing the value of particle volume friction and angle of wedge. On the other hand, the velocity profile increases by increasing the values of mixed convection $\lambda$ and suction (or injection) parameter. The temperature of nanofluid increases by increasing the volume friction. The temperature decreases due to enhancement in the effect of mixed convection parameter, suction (or injection) parameter, and angle of wedge. The lowest velocity and highest temperature of nanofluid are caused by the needle-shaped particles. The highest velocity and lowest temperature are determined by sphere particles. It is perceived that the entropy generation increases near the surface and then decreases and finally approaches zero near to free stream region. The maximum entropy production is found by the needle-shaped particles when it compared with the rest of shapes. The wall shear stress declines by increasing the size of particles. The minimum wall shear stress is seen for sphere-shaped particle and HFE-7500 base fluid. With the increase in volume friction and at small size of particle, the behavior of heat transfer rate increases. The maximum heat transfer is obtained when one chose disk-shaped nanoparticles and HFE-7500 fluid as compared to other. It is also found that small size particle and non-spherical shape are more stimulated for heat transfer rate.

Open Access This article is distributed under the terms of the Creative Commons Attribution 4.0 International License (http:// creativecommons.org/licenses/by/4.0/), which permits unrestricted use, distribution, and reproduction in any medium, provided you give appropriate credit to the original author(s) and the source, provide a link to the Creative Commons license, and indicate if changes were made.

\section{References}

Akbar NS, Nadeem S (2013) Peristaltic flow of a micropolar fluid with nano particles in small intestine. Appl Nanosci 3:461-468

Butt AS, Ali A (2013) Thermodynamical analysis of the flow and heat transfer over a static and a moving wedge. ISRN Thermodyn 2013:6-12

Cebeci T, Bradshaw P (1984) Physical and computational aspects of convective heat transfer. Springer, New York, p 174 
Choi SUS (1995) Enhancing thermal conductivity of fluids with nanoparticles. In: Siginer DA, Wang HP (eds) Developments and applications of non-Newtonian flows, FED, vol 231. ASME, New York, pp 99-105

Choi SUS, Zhang ZG, Yu W, Lockwood FE, Grulke EA (2001) Anomalous thermal conductivity enhancement in nanotube suspensions. Appl Phys Lett 79:2252-2254

Chon CH, Kihm KD, Lee SP, Choi SUS (2005) Empirical correlation finding the role of temperature and particle size for nanofluid $\mathrm{Al}_{2} \mathrm{O}_{3}$ thermal conductivity enhancement. Appl Phys Lett 87:531071-531073

Das SK, Putra N, Thiesen P, Roetzel W (2003) Temperature dependence of thermal conductivity enhancement for nanofluids. J Heat Transf Trans ASME 125:567-574

Eastman JA, Choi SUS, Yu W, Thompson LJ (2001) Anomalously increased effective thermal conductivity of ethylene glycolbased nanofluids containing copper nanoparticles. Appl Phys Lett 78:718-720

Eastman JA, Phillpot SR, Choi SUS, Keblinski P (2004) Thermal transport in nanofluids. Annu Rev Mater Res 34:219-246

Ellahi R (2013) The effects of MHD and temperature dependent viscosity on the flow of non-Newtonian nanofluid in a pipe: analytical solutions. Appl Math Model 37:1451-1457

Falkner VM, Skan SW (1931) Some approximate solutions of the boundary layer equations. Philos Mag 12(80):865-896

Fan J, Wang L (2011) Review of heat conduction in nanofluids. J Heat Transf 133:0408011-0408014

Ganapathirao M, Ravindran R, Pop I (2013) Non-uniform slot suction (injection) on an unsteady mixed convection flow over a wedge with chemical reaction and heat generation or absorption. Int $\mathrm{J}$ Heat Mass Transf 67:1054-1061

Ibrahim A, El-Amin MF, Salama A (2009) Effect of thermal dispersion on free convection in a fluid saturated porous medium. Int J Numer Methods Heat Fluid Flow 3:229-236

Kafoussias NG, Nanousis ND (1997) Magnetohydrodynamic laminar boundary-layer flow over a wedge with suction or injection. Can J Phys 75:733-745

Kafoussias N, Xenos M (2000) Numerical investigation of twodimensional turbulent boundary-layer compressible flow with adverse pressure gradient and heat and mass transfer. Acta Mech 141:201-223

Keblinski P, Prasher R, Eapen J (2008) Thermal conductance of nanofluids: is the controversy over? J Nanopart Res 10:1089-1097

Khandekar S, Joshi YM, Mehta B (2008) Thermal performance of closed two-phase thermosyphon using nanofluids. Int $\mathrm{J}$ Therm Sci 47:659-667

Kleinstreuer C, Feng Y (2011) Experimental and theoretical studies of nanofluid thermal conductivity enhancement: a review. Nanoscale Res Lett 6:229

Kumari M, Takhar HS, Nath G (2001) Mixed convection flow over a vertical wedge embedded in a highly porous medium. Heat Mass Transf 37:139-146

Kuo BL (2005) Heat transfer analysis for the Falkner-Skan wedge flow by the differential transformation method. Int $\mathrm{J}$ Heat Mass Transf 48:5036-5046

Lee D (2007) Thermophysical properties of interfacial layer in nanofluids. Langmuir 23:6011-6018

Lee S, Choi SUS, Li S, Eastman JA (1999) Measuring thermal conductivity of fluids containing oxide nanoparticles. J Heat Transf Trans ASME 121:280-289

Lee D, Kim JW, Kim BG (2006) A new parameter to control heat transport in nanofluids: surface charge state of the particle in suspension. J Phys Chem B 110:4323-4328

Liao SJ (2003) Beyond perturbation: Introduction to homotopy analysis method. Chapman \& Hall, Boca Raton
Liao S (2012) Homotopy analysis method in nonlinear differential equations. Higher Education Press, Beijing

Nadeem S, Mehmood R, Akbar NS (2013) Nanoparticle analysis for non-orthogonal stagnation point flow of a third order fluid towards a stretching surface. J Comput Theor Nanosci 10:2737-2747

Nadeem S, Maraj EN, Akbar NS (2014) Investigation of peristaltic flow of Williamson nano fluid in a curved channel with compliant walls. Appl Nanosci 4:511-521

Othman MIA, Zaki SA (2004) Thermal relaxation effect on magnetohydrodynamic instability in a rotating micropolar fluid layer heated from below. Acta Mech 17:187-197

Othman MIA, Hasona WM, Eraki EEM (2014) The effect of initial stress on generalized thermoelastic medium with three-phase-lag model under temperature dependent properties. Can J Phys 92(5):448-457

Patel HE, Das SK, Sundararajan T, Nair AS, George B, Pradeep T (2003) Thermal conductivities of naked and monolayer protected metal nanoparticle based nanofluids: manifestation of anomalous enhancement and chemical effects. Appl Phys Lett $83: 2931-2933$

Rao Y (2010) Nanofluids: stability, phase diagram, rheology and applications. Particuology 8:549-555

Shalkevich N, Escher W, Buergi T, Michel B, Si-Ahmed L, Poulikakos D (2010) On the thermal conductivity of gold nanoparticle colloids. Langmuir 26:663-670

Sheikholeslami M, Gorji-Bandpy M, Ganji DD, Rana P, Soleimani S (2014a) Magnetohydrodynamic free convection of $\mathrm{Al}_{2} \mathrm{O}_{3}$-water nanofluid considering thermophoresis and Brownian motion effects. Comput Fluids 94:147-160

Sheikholeslami M, Gorji-Bandpy M, Ganji DD (2014b) Lattice Boltzmann method for MHD natural convection heat transfer using nanofluid. Powder Technol 254:82-93

Sheikholeslami M, Gorji-Bandpy M, Ganji DD, Soleimani S (2014c) Heat flux boundary condition for nanofluid filled enclosure in presence of magnetic field. J Mol Liq 193:174-184

Sheikholeslami M, Ellahi R, Ashorynejad HR, Domairry G, Hayat T (2014d) Effects of heat transfer in flow of nanofluids over a permeable stretching wall in a porous medium. J Comput Theor Nanosci 11:486-496

Singh D, Timofeeva E, Yu W, Routbort J, France D, Smith D, Lopez-Cepero J (2009) An investigation of silicon carbidewater nanofluid for heat transfer applications. J Appl Phys 15:064306

Timofeeva EV, Routbort JL, Singh D (2009) Particle shape effects on thermophysical properties of alumina nanofluids. J Appl Phys 106:014304

Vafai K (2011) Porous media: applications in biological systems and biotechnology. Taylor \& Francis, New York

Vafai K, Kim SJ (1989) Forced convection in a channel filled with porous medium-an exact solution. J Heat Transf ASME 111:1103-1106

Watanabe T, Funazaki K, Taniguchi H (1994) Theoretical analysis on mixed convection boundary layer flow over a wedge with uniform suction or injection. Acta Mech 105:133-141

Xie H, Wang J, Xi T, Liu Y, Ai F, Wu Q (2002a) Thermal conductivity enhancement of suspensions containing nanosized alumina particles. J Appl Phys 91:4568-4572

Xie H, Wang J, Xi T, Liu Y (2002b) Thermal conductivity of suspensions containing nanosized SIC particles. Int J Thermophys 23:571-580

Xie H, Lee H, Youn W, Choi SUS (2003) Nanofluids containing multiwalled carbon nanotubes and their enhanced thermal conductivities. J Appl Phys 94:4967-4971

Xuan Y, Li Q (2000) Heat transfer enhancement of nanofluids. Int J Heat Fluid Flow 21:58-64 
Yih KA (1998) Uniform suction/blowing effect on force convection about wedge: uniform heat flux. Acta Mech 128:173-181

Yu W, Choi SUS (2004) The role of interfacial layers in the enhanced thermal conductivity of nanofluids: a renovated HamiltonCrosser model. J Nanopart Res 6:355-361

Yu DM, Routbort JL, Choi SUS (2008) Review and comparison of nanofluid thermal conductivity and heat transfer enhancements. Heat Transf Eng 29:432-460
Zhao Y, Liao S (2013) HAM-based package BVPh 2.0 for nonlinear boundary value problems. In: Liao S (ed) Advances in homotopy analysis method. World Scientific Press, Singapore 